\title{
Unsteady Behaviour of the Effervescent Atomizer
}

\author{
Marek Mlkvik1,* \\ ${ }^{1}$ Slovak University of Technology in Bratislava, Faculty of Mechanical Engineering, Nam. Slobody \\ 17, 812 31, Bratislava, Slovak Republic
}

\begin{abstract}
The effervescent atomizer is a well-established type of the twin-fluid nozzle with internal mixing of fluids. It is popular for the ability to process highly viscous liquids, such as liquid fuels, into a fine spray with low gas consumption. This study aims to investigate the performance of the effervescent nozzle when spraying the liquids with a viscosity up to $308 \mathrm{mPa} \cdot \mathrm{s}$. The working parameters of the nozzle were defined by the mass flows ratio of the gas to the liquid (GLR $=2.5$ to $20 \%$ ) and the gas pressure at the nozzle inlet $(\Delta p=0.14 \mathrm{MPa})$. The spray quality was investigated by the laser diffraction system, measuring the spray drop sizes. The investigated nozzle was able to atomize all of the model liquids. However, the liquid viscosity increase led to the need to operate the nozzle with the larger gas consumption. The minimum GLR for the spraying of the liquid with the viscosity $308 \mathrm{mPa} \cdot \mathrm{s}$ was $10 \%$, while the less viscous liquid $(60 \mathrm{mPa} \cdot \mathrm{s})$ was processed with the GLR $=2.5 \%$. It was observed that the spray quality was, at the low GLRs, lowered by unstable nozzle work, caused by the presence of the plug flow in the mixing chamber of the atomizer.
\end{abstract}

\section{Introduction}

The liquid atomization is an important physical process that is used in many industrial applications such as spray drying, irrigation, spray painting, liquid fuel combustion and many others [1-5]. The need to atomize the highly viscous liquids led to the development of the pneumatic atomizers, also known as the twin fluid atomizers. These devices use the energy of the pressurized gas to improve the liquid atomization process. The energy-saving operation of the twin-fluid nozzles demands reduction of the atomizing gas consumption. This demand is best fulfilled by the twin fluid atomizers with internal mixing of fluids (IMTFA).

The technological demands of most industrial applications are related to the drop sizes of the produced spray. The typical requirement of the spray drying process is the small size of produced drops. Irrigation and other agricultural processes need the specific drop size spectrum to avoid blowing off the liquid by the wind. The fuel combustion demands the presence of small drops as well as the large ones to maintain the stable burning process.

\footnotetext{
* Corresponding author: marek.mlkvik@stuba.sk
} 
The common demand of all the processes is the energy-saving operation which can be achieved by the twin-fluid atomizers only by the reduction of gas consumption.

The crucial part of the IM-TFA is the mixing chamber, where the two-phase flow develops. At ideal conditions is the internal two-phase flow pattern homogeneous, which positively influences the quality of produced spray. Inhomogeneous internal flow leads to the nozzle work, characterized by the unstable process of the liquid atomization. The spray quality is usually low at these conditions. Spray quality can be improved by the increase of the gas amount in the mixture or by the proper design of the atomizer. The effervescent atomizer, investigated in this study, is a well-established device with the mixing chamber designed to create small gas bubbles dispersed in the liquid $[2,6]$.

This study aims to test the ability of the effervescent atomizer to perform the atomization of the viscous liquids at the very low gas consumptions. We will analyse the spray quality and stability to quantify the nozzle performance. The spray stability, in this study, is defined as the operation of the nozzle, with stable conditions of the liquid breakup. The result of the stable work of the nozzle is a spray with uniform sizes of the produced drops. The drop sizes spectrum and the average drop sizes will be, for purposes of this study, the qualitative criteria of the spray. The spray quality and spray stability will be related to the internal two-phase flow. The results will be used to define the range of working parameters at which the atomizer provides satisfactory performance.

\section{Experiment}

A simplified schematic of the test rig is shown in Figure 1. The atomizer (Figure 2) was operated at a constant overpressure of the air at the mixing chamber inlet $(\Delta p=0.14 \mathrm{MPa})$ which was maintained by the reducing valve placed in the air supply line. The liquid flow, provided by the eccentric screw pump, was controlled by the bypass valve. This control components configuration was chosen to allow independent control of the nozzle working parameters: mixing chamber overpressure and the gas to the liquid mass flows ratio. The model liquids were aqueous solutions of the maltodextrin with different concentrations and physical properties (Table 1.)

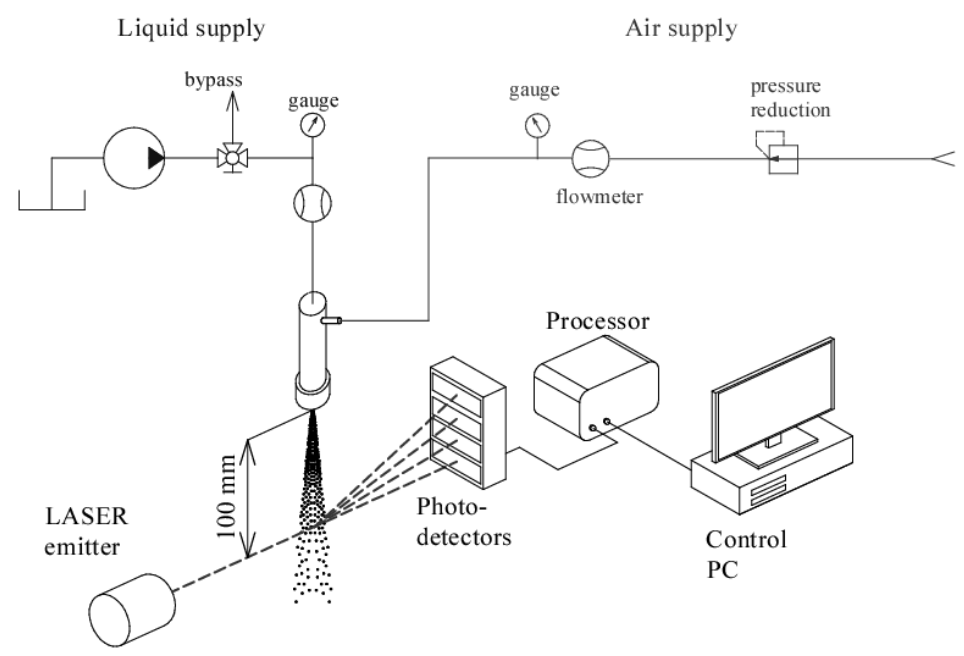

Fig. 1. Test bench with the optical measurement system. 
Table 1. Physical properties of the model liquids.

\begin{tabular}{|c|c|c|c|}
\hline Liquid name & $\boldsymbol{\mu}[\mathbf{m P a} \cdot \mathbf{s}]$ & $\boldsymbol{\sigma}[\mathbf{N} / \mathbf{m}]$ & $\boldsymbol{\rho}\left[\mathbf{k g} / \mathbf{m}^{\mathbf{3}}\right]$ \\
\hline MD40 & 60 & 0.074 & 1183 \\
\hline MD50 & 308 & 0.076 & 1241 \\
\hline
\end{tabular}

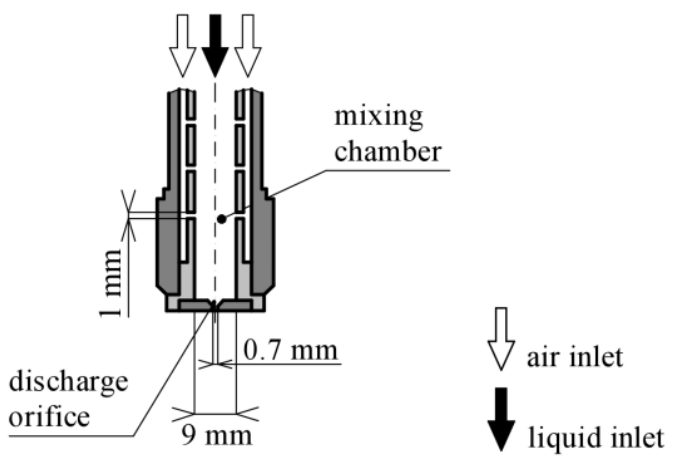

Fig. 2. Schematic drawing of the used effervescent nozzle.

The drop sizes were measured by the Malvern Spraytec laser diffraction system and it was characterized by the instantaneous value of the Sauter mean diameter $\left(D_{32}\right.$, t.d. $)$. The measurement point was placed $100 \mathrm{~mm}$ downstream the exit orifice. This distance was chosen to ensure that the spray did not contain liquid ligaments or non-spherical drops. The drop sizes measurement frequency was $500 \mathrm{~Hz}$, and the measurement period was $25 \mathrm{~s}$ long. The measured $D_{32}$, t.d. values were further converted into the Cumulative Distribution Function (CDF). The shapes of the CDF curves were analyzed and related to spray stability.

The internal two-phase flows as the potential source of the spray pulsations were investigated indirectly, using the two-phase flow maps. The first of the maps was wellestablished and widely used Baker's map [7] created from the experimental data. The second map was a theoretical diagram established by Barnea [8]. Both the maps were used in a version for the vertical flow. The results provided by the two-phase flow maps were confronted with the measured spray characteristics to explain the origin of the spray stability or instability.

\section{Results}

At first was tested the atomizers' ability to spray the liquid at various gas consumption regimes (Table 2.). The nozzle sprayed the less viscous liquid within the whole range of the GLRs. The more viscous liquid was possible to spray only with the GLR $=10$ and $20 \%$.

Table 2. Spraying ability of the nozzle for different GLRs and liquids.

\begin{tabular}{|c|c|c|c|c|}
\hline $\boldsymbol{\mu}[\mathbf{m P a} \cdot \mathbf{s}]$ & \multicolumn{5}{|c|}{ GLR [\%] } \\
\hline & $\mathbf{2 . 5}$ & $\mathbf{5}$ & $\mathbf{1 0}$ & $\mathbf{2 0}$ \\
\hline 60 & $\mathrm{~A}$ & $\mathrm{~A}$ & $\mathrm{~A}$ & $\mathrm{~A}$ \\
\hline 308 & $\mathrm{X}$ & $\mathrm{X}$ & $\mathrm{A}$ & $\mathrm{A}$ \\
\hline
\end{tabular}

Legend: A - Atomizer sprayed the liquid, X-Atomizer did not spray the liquid 


\subsection{Nozzle performance analysis}

The results of the drop sizes measurement in Figure 3, 4 indicate that the nozzle produced, at GLR $=20 \%$, spray of comparable average drop size about $40 \mu \mathrm{m}$. The viscosity of liquid had a low or negligible effect on the spray mean size, which is in accordance with the previous works [2]. The spread of the drop sizes from the mean values was also comparable which is documented by the narrow CDF curves (Figure 5b, Figure 6). The decrease of GLR to the value $10 \%$ did not significantly influence the spraying of the less viscous liquid which is documented by the almost identical CDF curves in Figure $5 \mathrm{~b}$. The MD50 was sprayed with the mean drop size close to $60 \mu \mathrm{m}$, which means the $30 \%$ increase of $D_{32}$, t.d. The $\mathrm{CDF}$ curve for GLR $=10 \%$ in Figure 6 is narrow almost within the whole range. The chart suggests that there were present about $5 \%$ of very large drops which decreased the spray quality. These drops were the result of the unstable work of the nozzle. Thus, not only the mean drop size increased but also the spray stability was influenced by the GLR reduction.

The spraying at GLR $=5$ and $2.5 \%$ was possible only with the MD40 as the effervescent nozzle did not process the more viscous liquid.

The atomizer provided, with MD40 at GLR $=5 \%$, satisfactory performance. The CDF curve (Figure 5a) was broader than for the previously investigated GLR regimes, what correspond to the observed drop size variations in Figure 3. Both the charts show that the drop size varied within the range between 45 to 100 microns. Only a small quantity of drops were larger, with a maximum size of $150 \mu \mathrm{m}$. Even when the spray quality, in terms of fine drops, got worsen (compared to the higher GLR regimes) the nozzle provided a performance, which suits certain technologies which require specific drop size range, rather than fine spray, like fuel burning.

Further reducing of the gas consumption to GLR $=2.5 \%$ had two negative effects on the atomizer's performance. The mean drop size increased up to $100 \mu \mathrm{m}$, which is a value that cannot be accepted in applications such as a spray drying where are the fine drops a key factor. The second effect were the bursts of the drops, several times larger than average, which can be observed in Figure 3. The CDF curve in Figure 5a also indicates a presence of the drops much smaller than was the average. The total range of the measured drop sizes varied from a minimum of $50 \mu \mathrm{m}$ to a maximum of $1000 \mu \mathrm{m}$.

The provided analysis had shown that the decrease of the spray quality was related to the unstable spraying process, usually observed at the low gas consumption regimes. The internal flow analysis will be provided in the next section to clarify the origins of the observed nozzle behaviour.

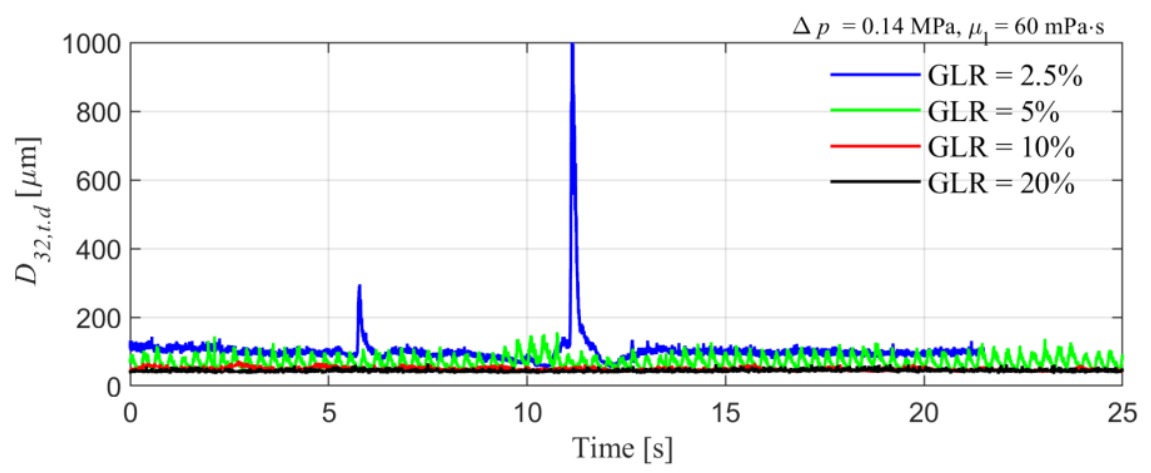

Fig. 3. Time record of the measured SMD for the less viscous liquid at GLR $=2.5$ and $5 \%$. 


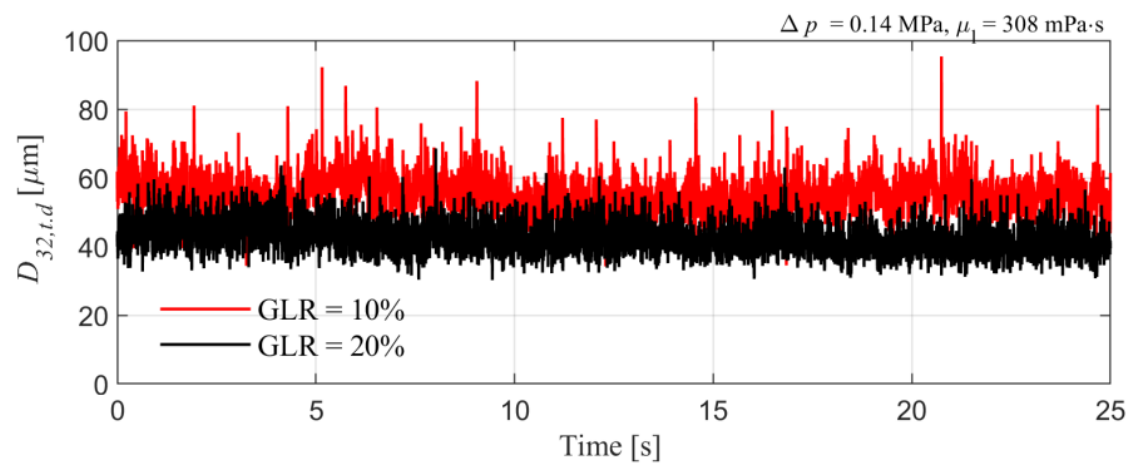

Fig. 4. Time record of the measured SMD for the most viscous liquid. Nozzle did not atomize the liquid at the lower GLRs.
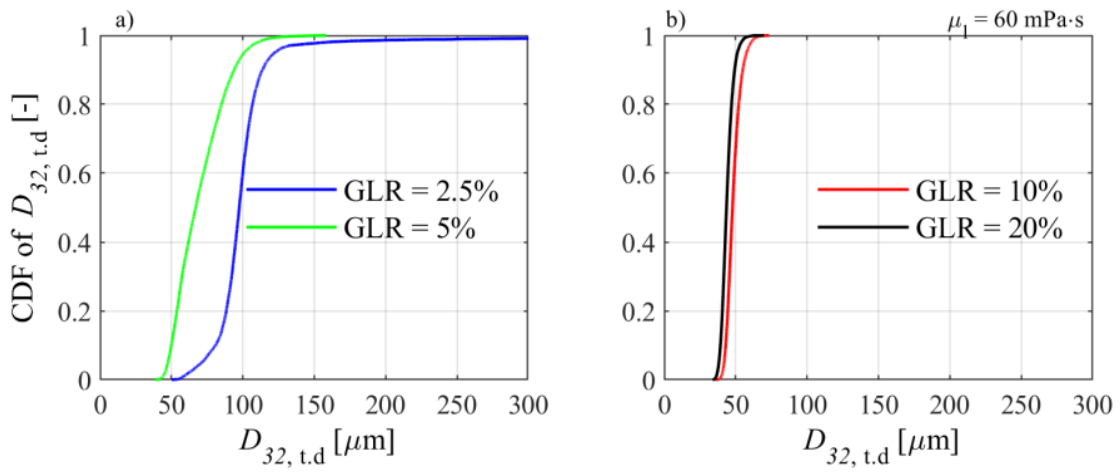

Fig. 5. CDF of time-dependent drop sizes for spraying the less viscous liquid.

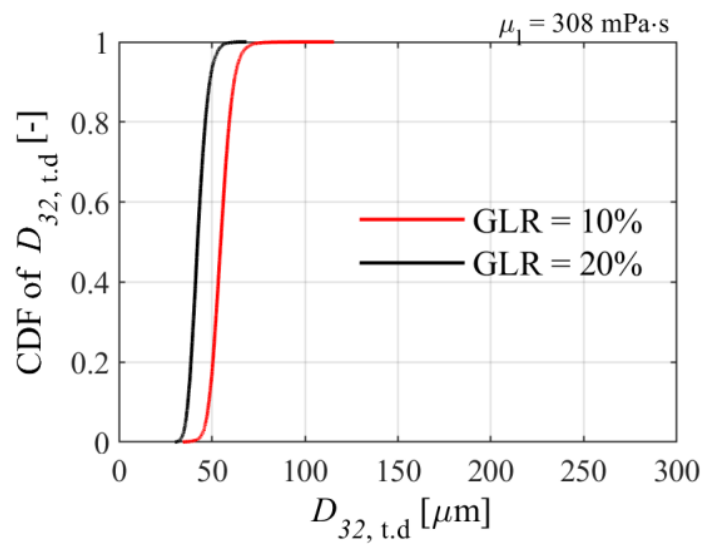

Fig. 6. CDF of time-dependent drop sizes for spraying the more viscous liquid. 


\subsection{Internal two-phase flows of the effervescent nozzle}

The atomization by the twin-fluid nozzles starts with the injection of the fluids into the mixing chamber. The mixture flows down the mixing chamber, it reaches the discharge orifice and it is ejected to the open atmosphere, where the spray is formed. This indicates, that the processes inside the mixing chamber play a crucial role in the liquid atomization by the effervescent atomizer. The original design goal of the effervescent atomizer was to decrease the gas consumption to the level, where the dispersed (bubbly) internal flow occurs. The bubbles in the liquid at this regime flow through the discharge orifice and then expand, which shatters the liquid [3]. This process produces a stable spray with the narrow spectrum of the drop sizes.

The performance of the tested nozzle indicate, that the bubbly flow was not present in the mixing chamber. We used the two-phase flow maps to estimate the internal two-phase flow pattern and to explain the observed nozzle pulsations. The estimated two-phase flow patterns are shown in Figure 7,8.

Both the regimes with high gas consumption (GLR $=20 \%$ and $10 \%$ ) were typical by the low spray pulsations which originated from the internal two-phase flow pattern. The used flow maps differ in the two-phase flow pattern estimation. The modified Baker's map estimated the plug or slug internal flow for all the regimes and both liquids. The Barnea's map predicted annular internal flow for the less viscous liquid $(\mu=60 \mathrm{mPa} \cdot \mathrm{s})$ and elongated bubbles regime for the liquid with $\mu=308 \mathrm{mPa} \cdot \mathrm{s}$. The results of the spray quality analysis suggest that the more precise estimation of the flow pattern was, for the less viscous liquid, provided by the Barnea's map. As the work [9] suggests, the annular internal flow results to the continuous passage of the thin liquid film through the discharge orifice and results in the fine spray with the narrow drop size spectrum which was observed in the spray. For the more viscous liquid, both the flow maps provided a comparable estimation of the two-phase flow (elongated bubbles and slug/plug flow pattern) which cause local variations of the mixture composition in the discharge orifice. The observed spray was stable with the narrow drop sizes spectrum, which suggests, that both the flow maps failed to estimate the internal flow correctly.

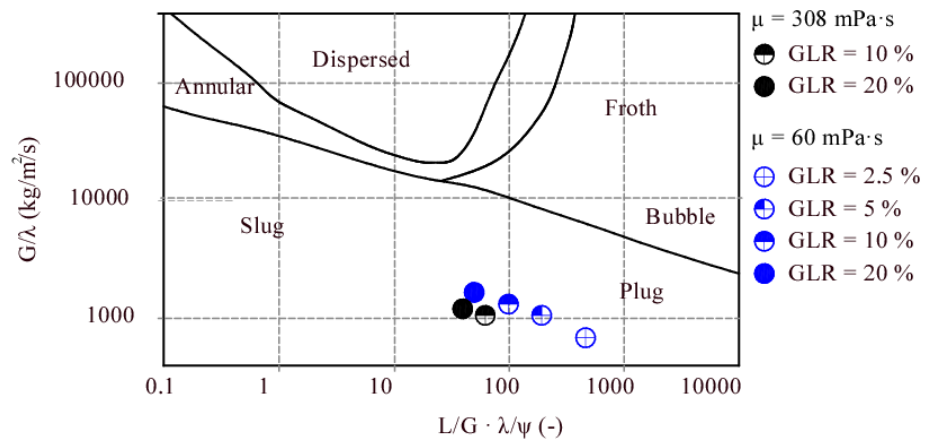

Fig. 7. Estimated internal flow regimes according to the Modified Baker's map [10].

For the working regimes with the low gas consumptions were the estimations of the internal flow pattern possible only for the less viscous liquid. Both the maps estimated a two-phase flow type, either plug flow or elongated bubbles, which resulted in the temporal variations in the mixture composition in the discharge orifice. Depending on the immediate liquid to gas ratio in the discharged mixture were in the primary breakup zone present different types of liquid structures. The large liquid structures were produced when the amount of atomizing gas decreased. They have further disrupted which led to the 
production of the large drops. The thin fibres and the small spherical drops were produced when the gas amount in the mixture was sufficient to break the liquid into smaller structures. Presence of all the described liquid structures at the GLR $=2.5 \%$ is shown in Figure 9.

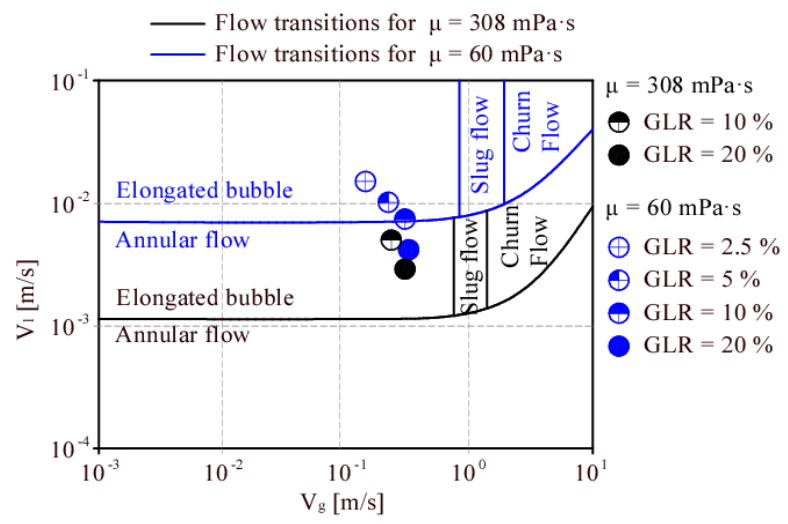

Fig. 8. Estimated internal flow regimes according to the Barnea's map.

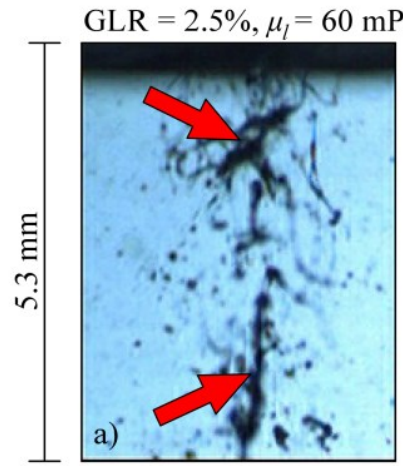

large liquid structures

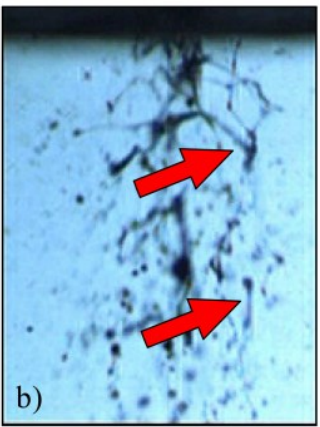

fibers and ligaments

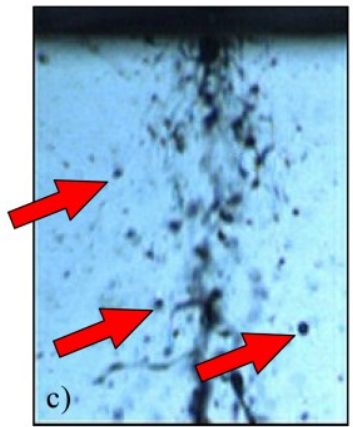

spherical drops

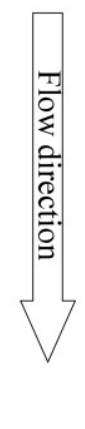

Fig. 9. The different liquid structures, observed in the discharge zone of the nozzle at the low GLR.

\section{Conclusion}

The presented paper was oriented to compare the work of the effervescent atomizer when spraying the two viscous liquids. The comparison was focused on the spray quality, defined by the size spectrum of produced drops.

The analysis has shown that the nozzle produced a spray of the acceptable quality with both the liquids at the regimes defined by the GLR $=10$ and $20 \%$. The atomization of both tested liquids, at these working conditions, was stable. This resulted in the production of the sprays with the narrow drop sizes spectrum.

The nozzle performance decreased when the GLR was $5 \%$ and $2.5 \%$. The atomizer did not spray the more viscous liquid and the spray quality significantly decreased with the less viscous liquid. The work of the nozzle was characterized by the presence of spray pulsations, resulting in a wider spectrum of the produced drops than for the high gas consumption regimes. 
The internal two-phase flow of the nozzle was identified as the source of the stable or unstable behaviour of the nozzle. The two-phase patterns of the flow in the mixing chamber were estimated by the different two-phase flow maps. The estimated two-phase flow patterns were compared with the observations of the spray stability. It was found that estimations from Baker's map were not in compliance with the experimental data. This map estimated the internal two-phase flow pattern as plug/slug for all the tested working regimes, even for those, which were characterized by the stable nozzle work without spray pulsations. The Barnea's map estimated the annular flow for the regimes, where the nozzle work was stable and the elongated bubbles flow for the pulsating regimes where the nozzle produced a wider spectrum of drop sizes.

The conclusion of the presented research is that the single nozzle could be used to process the various liquids within the wide viscosity range. However, spraying of the more viscous liquids with the same operating pressure requires to increase the gas consumption. The stability of work of the effervescent nozzle is possible to estimate for different liquids and working parameters by the two-phase flow maps. However, not all the available two-phase flow maps are suitable for this purpose. In the presented paper, the Barnea's map was found to provide an acceptable flow internal two-phase flow pattern estimation.

\section{References}

1. J. Jedelsky, M. Jicha, Fuel 2013, 111, 836-844 (2013)

2. G. Ferreira, J. Garcia, F. Barreras, A. Lozano, E. Lincheta, Fuel Process. Technol., 90, 270-278 (2009)

3. H. N. Buckner, P. E. Sojka, A. H. Lefebvre, ASME Publ. Pet. Div., 30, 105-108 (1990)

4. S. G. Daviault, O. B. Ramadan, E. A. Matida, P.M. Hughes, R. Hughes, Fuel, 98, 183-193 (2012)

5. L. Broniarz-Press, M. Ochowiak, S. Woziwodzki, Int. J. Heat Fluid Flow, 31, 651658 (2010)

6. J. Chin, A. Lefebvre, J. Eng. Gas Turbines Power, 117, 266-271 (1995)

7. O. Baker, Oil Gas J., 53, 185-195 (1954)

8. D. Barnea, O. Shoham, Y. Taitel, Chem. Eng. Sci., 37, 735-740 (1982)

9. M. Lorcher, F. Schmidt, D. Mewes, In: 9th ICLASS, pp. 12-9 (2003)

10. P. B. Whalley, Oxford University Press, 291 p., ISBN 0198561814 (1987) 